"C 2019 IEEE. Personal use of this material is permitted. Permission from IEEE must be obtained for all other uses, in any current or future media, including reprinting/republishing this material for advertising or promotional purposes, creating new collective works, for resale or redistribution to servers or lists, or reuse of any copyrighted component of this work in other works." 


\title{
Enhanced AoA Estimation Using Localized Hybrid Dual-Polarized Arrays
}

\author{
Hang Li, Thomas Q. Wang, Xiaojing Huang, and J. Andrew Zhang \\ Global Big Data Technologies Center (GBDTC), University of Technology Sydney, Australia \\ Emails: \{Hang.Li, Qian.Wang, Xiaojing.Huang, Andrew.Zhang\}@uts.edu.au
}

\begin{abstract}
With balanced system performance, implementation complexity and hardware cost, hybrid antenna array is regarded as an enabling technology for massive multiple-input and multiple-output communication systems in millimeter wave (mmWave) frequencies. Angle-of-arrival (AoA) estimation using a localized hybrid array faces the challenges of the phase ambiguity problem due to its localized nature of array structure and susceptibility to noises. This paper discusses AoA estimation in an mmWave system employing dual-polarized antennas. We propose an enhanced AoA estimation algorithm using a localized hybrid dual-polarized array for a polarized mmWave signal. First, the use of dual-polarized arrays effectively strengthens the calibration of differential signals and resulting signal-tonoise ratio with coherent polarization combining, leading to an enhanced estimate of the phase offset between adjacent subarrays. Second, given the phase offset, an initial AoA estimate can be obtained, which is used to update the phase offset. By employing the updated one, the AoA is re-estimated with improved accuracy. The closed-form mean square error (MSE) lower bounds of AoA estimation are derived and compared with simulated MSEs. The simulation results show that the proposed algorithm in combination with hybrid dual-polarized arrays significantly improves the estimation accuracy compared with the state of the art.
\end{abstract}

Index Terms-Hybrid dual-polarized array, localized subarrays, angle-of-arrival estimation, polarization diversity and mmWave communications.

\section{INTRODUCTION}

Millimeter wave (mmWave) hybrid antenna array is known as a highly promising technique to achieve great balance between performance and cost for future cellular communications [1]-[4]. Unlike the microwave channels characterized by rich scattering, the line-of-sight propagation dominates the mmWave channels. Therefore, its angle-of-arrival (AoA) acquisition is of great concern for signal reception at the receiver array. Particularly when receiving a polarized signal, the use of a single-polarized hybrid array often incurs the received signal power loss and thus poor AoA estimation accuracy because of the polarization mismatch between the receiving antennas and the incoming wave. As a result, hybrid dual-polarized array [5]-[7] is regarded as an effective solution to immunizing the degradation of signal-to-noise ratio (SNR) and improving the AoA estimation performance.

A hybrid antenna array typically consists of multiple analog subarrays with phase-tunable antenna elements. It is classified into two types of regular configurations, i.e., localized and

This research was supported under Australian Research Council's Discovery Projects funding scheme (project number DP160101693). interleaved arrays in terms of antenna distribution in a subarray [4]. The localized array is easier to build and form a larger array with multiple modules in feeding networks, and thus is more practical in terms of hardware feasibility. The AoA estimation using a localized array has been studied in [8]-[13]. All of them exploit the cross correlations between consecutive subarrays to extract the AoA information, and seek to solve the phase ambiguity problem. The phase ambiguity results from an unknown integer multiple of $2 \pi$ difference between $N$ times of an AoA information parameter (i.e., $N u$ ) and the argument of the cross correlations, where $N$ is the number of antennas in a subarray and $u$ denotes the AoA information parameter. The works in [8]-[10] leverage the same phase shift configuration across different subarrays for constructive accumulation of cross correlations. In [8], a differential beam search algorithm is proposed to search all possible beams and select the estimate with the maximum output power, which however introduces a long estimation delay. An adaptive searching and tracking algorithm is then developed in [9] to speed up the searching process. To avoid this delay, a frequency-domain AoA estimation algorithm applied to a wideband array is proposed in [10], whereas the noise induced by the conjugate product of cross correlations is greatly amplified.

The authors in [11] propose a novel subarray-specific phase shift configuration to remove the ambiguity by directly estimating $u$. Its basic idea is that $N u$ is firstly estimated by calibrating the signs of cross correlations and combining them constructively. By suppressing $e^{j m N u}$ in the $m$ th subarray output signal, one can take its inverse discrete Fourier transform (IDFT), and then calculate the correlations of the Fourier coefficients to unambiguously estimate $u$. Furthermore, the authors in [12], [13] generalize the phase shift designs of [11] to narrowband and wideband systems respectively, and find the cross correlations with the same signs except the greatest one. This improves the calibration accuracy of cross correlations and thus coherent combining for estimating $\mathrm{Nu}$. Without consideration of the polarization, all of the above works only study the AoA estimation using a single-polarized hybrid array. However, in practice, the reception of a polarized wave has a significant impact on AoA estimation accuracy due to polarization mismatch. Therefore, we propose to employ hybrid dual-polarized arrays for the AoA estimation of a polarized wave.

In this paper, we study the reception of a polarized mmWave signal using a localized hybrid dual-polarized array. It is shown 


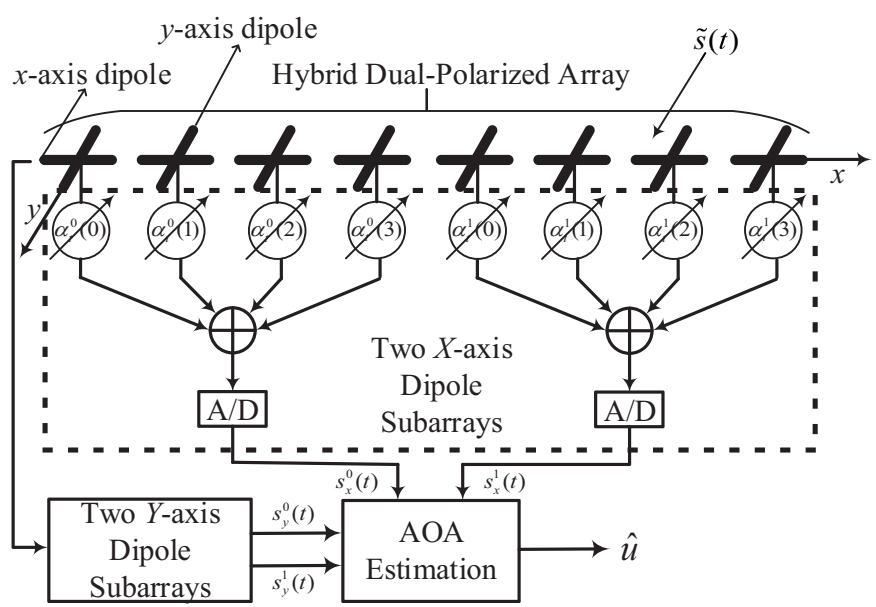

Fig. 1. Illustration of a localized dual-polarized array with two subarrays.

that exploiting dual-polarized arrays can efficiently align the signs of differential signals and thus enhance the SNR of $N u$ estimation with polarization combining. An enhanced AoA estimation algorithm using subarray-specific time-varying phase shifts is proposed accordingly, where the AoA information, $u$, is re-estimated by updating the estimate of $N u$. In the context of both dual-polarized and single-polarized arrays, we also derive the closed-form mean square error lower bounds (MSELBs) for the AoA estimation, respectively. Simulation results demonstrate that the proposed algorithm together with hybrid dual-polarized arrays outperforms the state of the art in terms of estimation accuracy.

\section{SYSTEM MODELS}

Consider a uniform linear hybrid dual-polarized array with $M$ localized subarrays, each having $N$ electromagnetic vector sensors (EMVS) [14]. Each EMVS includes two spatially collocated orthogonal dipoles, denoted by $x$-axis and $y$-axis dipoles respectively, which are used to measure the components of the incoming electric field projected onto the directions of $x$ - and $y$-axes. As a result, two replicas of the signals collected from all the $x$ - and $y$-axis dipoles can be obtained respectively for subsequent signal processing. Prior to coherent combining of the received signals from dual dipoles, each of them is separately and identically processed in analog and digital domains. An example of a dual-polarized array with two localized subarrays and the associated signal processing modules are illustrated in Fig. 1, where the radio frequency and down conversion modules are omitted for simplicity. Note that the module named "Two $y$-axis Dipole Subarrays" enclosed in the solid box has the same components with the one named "Two $x$-axis Dipole Subarrays" shown in the dashed box.

Consider the reception of a transverse electromagnetic wave signal $\tilde{s}(t)$ with elevation angle $\theta$ and polarization state $(\gamma, \eta)$ produced by its electric field [14]. $\gamma \in\left[0, \frac{\pi}{2}\right)$ and $\eta \in[-\pi, \pi)$ represent the auxiliary polarization angle and the polarization phase difference, respectively. For example, $\eta=0$ refers to linearly-polarized waves, while $\gamma=\frac{\pi}{4}$ and $\eta= \pm \frac{\pi}{2}$ refer to left/right circularly-polarized wave. As a result, the electric field vector $\mathbf{e}$ is expressed in Cartesian coordinates as $\mathbf{e}=e_{x} \mathbf{v}_{x}+e_{y} \mathbf{v}_{y}+e_{z} \mathbf{v}_{z}$, where $\mathbf{v}$ is a unit vector along the subscript's coordinate, and $\left[e_{x}, e_{y}, e_{z}\right]=\left[\sin \gamma \cos \theta e^{j \eta}, \cos \gamma,-\sin \gamma \sin \theta e^{j \eta}\right]$ denote the responses of the corresponding subscripts. After down-conversion and analog-to-digital (A/D) conversion, the received signal through the $m$ th subarray, $m=0, \ldots, M-1$, can be expressed as

$$
\begin{aligned}
{\left[s_{x}^{m}(t), s_{y}^{m}(t)\right]=} & {\left[e_{x}, e_{y}\right] \cdot \tilde{s}(t) P_{t}^{m}(u) e^{j m N u} } \\
& +\left[n_{x}^{m}(t), n_{y}^{m}(t)\right],
\end{aligned}
$$

where $s_{x}^{m}(t)$ and $s_{y}^{m}(t)$ denote the signals received by $x$ - and $y$-axis dipoles respectively, and $P_{t}^{m}(u)$ is the radiation pattern of the $m$ th subarray at the $t$ th symbol, expressed as

$$
P_{t}^{m}(u)=\sum_{n=0}^{N-1} \check{P}_{n}^{m}(u) e^{j\left(n u+\alpha_{t}^{m}(n)\right)},
$$

with $\check{P}_{n}^{m}(u)$ denoting the radiation pattern of the $n$th $x$-/y-axis dipole $(n=0, \ldots, N-1)$ at the $m$ th subarray. As in [11], [12], we assume that $\check{P}_{n}^{m}(u)=1$ in this paper. $\alpha_{t}^{m}(n)$ denotes the corresponding phase shift of the $n$th EMVS and $u=\frac{2 \pi}{\lambda} d \sin \theta$. $\lambda$ is the wavelength of the carrier, and $d$ denotes the spacing between adjacent EMVS. $\left[n_{x}^{m}(t), n_{y}^{m}(t)\right]$ are the independent complex additive white Gaussian noises corresponding to $x$ and $y$-axis dipoles at the outputs of the $m$ th subarray with the same power $\sigma_{n}^{2}$.

\section{Enhanced AOA Estimation Algorithm}

In this section, we apply the phase shift designs in [12] to hybrid dual-polarized arrays to obtain an estimate of $N u$, which is then used to suppress $e^{j m N u}$ in the output signal of the $m$ th subarray followed by the estimation of $u$.

\section{A. Estimation of $\mathrm{Nu}$}

As in [12], the $n$th $(n=0, \ldots, N-1)$ phase shift of the $m$ th $(m=0, \ldots, M-1)$ subarray at the $t$ th $(t=0, \ldots, T-1)$ symbol is given by

$$
\alpha_{t}^{m}(n)=-n \alpha_{t}^{m}=\frac{-2 \pi n(\bmod \{m, K\} T+t)}{L},
$$

where $\alpha_{t}^{m}$ denotes the phase shift difference between any two adjacent EMVS of the $m$ th subarray at the $t$ th symbol, which indicates that each subarray directs at a predefined direction; $K$ is the number of different phase shifts for any symbol. Specifically, $K \in(2, M]$ and $N=Q K$, where $Q$ is an integer; $T$ is the number of reference signals; $L=T K$ is the overall number of different phase shifts adopted in the system. The configuration given by (3) enables the array to sweep $L$ evenly distributed directions within $[-\pi, \pi)$, thereby guaranteeing AoA acquisition using at least one of the $L$ beams with high gain; $\bmod \{\cdot, \cdot\}$ stands for the modulo operation, and thus $\bmod \{m, K\}$ implies that the cycle of $\alpha_{t}^{m}$ occurs every $K$ subarrays in a symbol. 
By substituting (3) into (2), one can rewrite it as

$$
P_{t}^{m}(u)=\sum_{n=0}^{N-1} e^{j n\left(u-\alpha_{t}^{m}\right)}=e^{j(N-1) \omega_{t}^{m}} \frac{\sin \left(N \omega_{t}^{m}\right)}{\sin \left(\omega_{t}^{m}\right)},
$$

where $\omega_{t}^{m}=\left(u-\alpha_{t}^{m}\right) / 2$. When the first $K$ subarrays are considered, $\omega_{t}^{m}$ can be simplified as $\omega_{t}^{m}=\frac{u}{2}-\pi\left(\frac{m}{K}+\frac{t}{L}\right)$. Calculating the differential signals between the output signals of the $m$ th and $(m+1)$ th subarrays generates

$$
\begin{aligned}
& {\left[\rho_{x}^{m}(t), \rho_{y}^{m}(t)\right] } \\
= & {\left[\left(s_{x}^{m}(t)\right)^{*} s_{x}^{m+1}(t),\left(s_{y}^{m}(t)\right)^{*} s_{y}^{m+1}(t)\right] } \\
= & \underbrace{\left[\left|e_{x}\right|^{2},\left|e_{y}\right|^{2}\right] \cdot|\tilde{s}(t)|^{2} G_{t}^{m}(u) e^{j N u}+\left[z_{x}^{m}(t), z_{y}^{m}(t)\right],}_{\text {signal component }}
\end{aligned}
$$

where

$$
\begin{aligned}
G_{t}^{m}(u) & =\left(P_{t}^{m}(u)\right)^{*} P_{t}^{m+1}(u) \\
& =e^{\frac{-j(N-1) \pi}{K}} \frac{\sin \left(N \omega_{t}^{m}\right) \sin \left(N \omega_{t}^{m+1}\right)}{\sin \left(\omega_{t}^{m}\right) \sin \left(\omega_{t}^{m+1}\right)} \\
& =e^{\frac{-j(N-1) \pi}{K}} \frac{(-1)^{Q} \sin ^{2}\left(N \omega_{t}^{m}\right)}{\sin \left(\omega_{t}^{m}\right) \sin \left(\omega_{t}^{m+1}\right)},
\end{aligned}
$$

$\left[z_{x}^{m}(t), z_{y}^{m}(t)\right]$ are approximated as the zero-mean complex Gaussian noises given by

$$
\begin{aligned}
z_{l}^{m}(t)= & \left(n_{l}^{m}(t)\right)^{*} n_{l}^{m+1}(t)+e_{l}^{*} \tilde{s}^{*}(t)\left(P_{t}^{m}(u)\right)^{*} e^{-j m N u} n_{l}^{m+1}(t) \\
& +e_{l} \tilde{s}(t) P_{t}^{m+1}(u) e^{j(m+1) N u}\left(n_{l}^{m}(t)\right)^{*}, \quad l \in\{x, y\}
\end{aligned}
$$

and $(\cdot)^{*}$ and $|(\cdot)|$ represent the conjugate and absolute value of $(\cdot)$, respectively. Eq. (5) shows that $\rho_{x}^{m}(t)$ and $\rho_{y}^{m}(t)$ have an identical phase, $N u$, in signal component, and their sum has their individual components added in phase.

It is also stated by the Theorem 1 [12] that among the terms, $G_{t}^{m}(u), m=0, \ldots, K-1$, only $G_{t}^{m^{\prime}}(u)$ with the largest amplitude has the opposite sign of the remainings. Therefore, we propose to find $m^{\prime}$ which leads to the largest amplitude of $\rho_{x}^{m}(t)+\rho_{y}^{m}(t)$, i.e.,

$$
m^{\prime}=\underset{m=0: K-1}{\operatorname{argmax}}\left\{\left|\rho_{x}^{m}(t)+\rho_{y}^{m}(t)\right|\right\} .
$$

Given $m^{\prime}$, the signs of the differential signals can be aligned following

$$
\left[\tilde{\rho}_{x}^{m}(t), \tilde{\rho}_{y}^{m}(t)\right]= \begin{cases}(-1)^{Q}\left[\rho_{x}^{m}(t), \rho_{y}^{m}(t)\right], & m \neq m^{\prime} \\ (-1)^{Q+1}\left[\rho_{x}^{m^{\prime}}(t), \rho_{y}^{m^{\prime}}(t)\right], & m=m^{\prime}\end{cases}
$$

to perform in-phase combination for the estimate of $N u, \widehat{N u}$. Specifically, we can combine $\tilde{\rho}_{x}^{m}(t)$ and $\tilde{\rho}_{y}^{m}(t)$ across all subarrays and symbols constructively to improve the accuracy of $\widehat{N u}$ as shown in Algorithm I, where $\arg \{\cdot\}$ denotes the argument of the complex number $(\cdot)$.

Note that, compared with Step 11 of Algorithm 1 in [12], Step 6 of our algorithm can enhance the ability of identifying the correct $m^{\prime}$ by in-phase combining the differential signals of EMVS, thus effectively suppressing the noise and indirectly improving the SNR of estimation. This will be verified in simulation results. Also, we exploit the coherent combining of the aligned differential signals from dual dipoles at Step 10 to directly achieve SNR gains over that in [12].

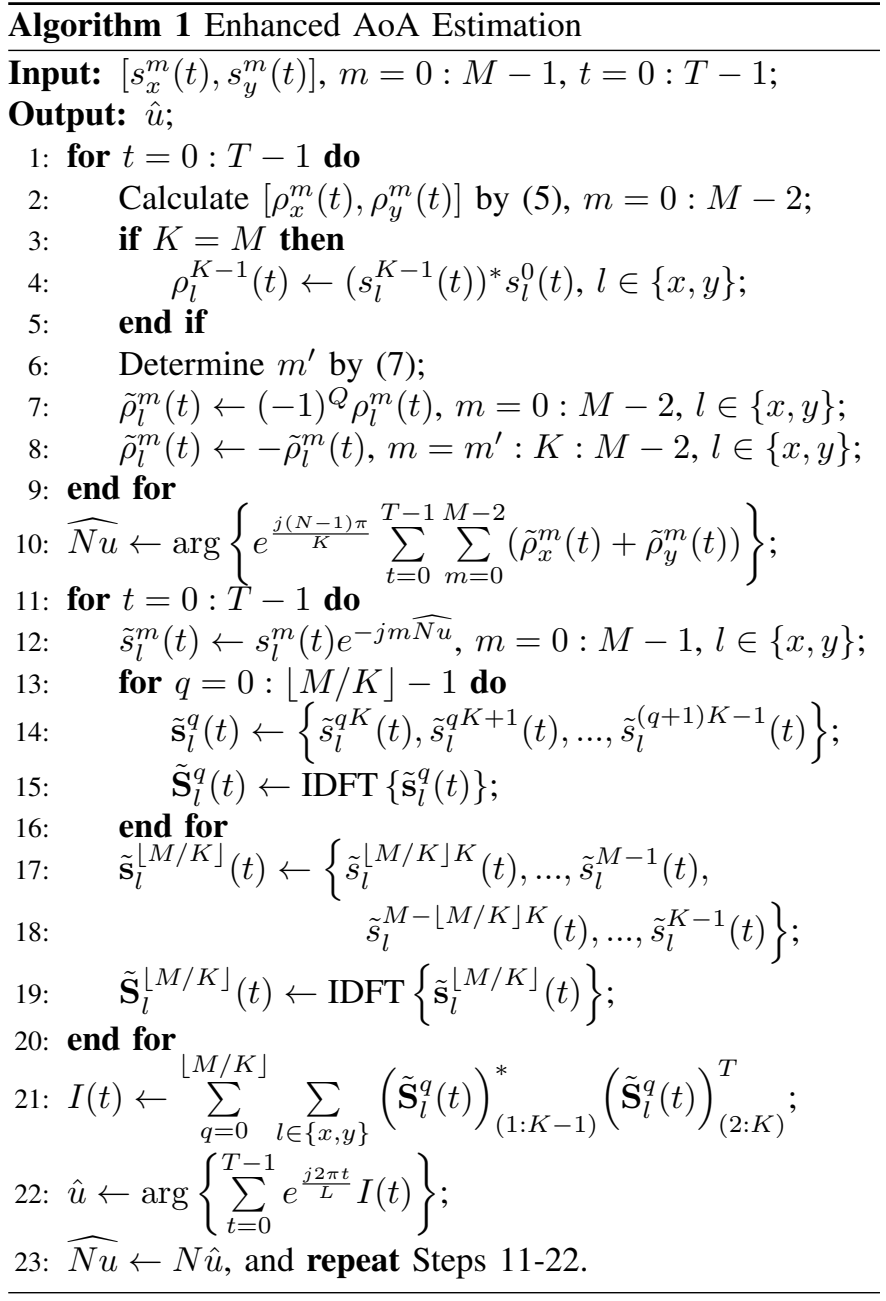

\section{B. Estimation of $u$}

Given $\widehat{N u}$, one can calibrate the output signals of subarrays, $\left[s_{x}^{m}(t), s_{y}^{m}(t)\right]$, by multiplying $e^{-j m \overline{N u}}$, i.e., $\left[s_{x}^{m}(t), s_{y}^{m}(t)\right] e^{-j m \widehat{N u}}$. Given that $e^{j m(N u-\widehat{N u})} \approx 1$, $\left[s_{x}^{m}(t), s_{y}^{m}(t)\right]$ can be nearly perfectly calibrated. Then, by performing the $K$-point IDFTs of $\left[s_{x}^{m}(t), s_{y}^{m}(t)\right] e^{-j m \widehat{N u}}$, one can produce $\left[\widetilde{S}_{x}^{k}(t), \widetilde{S}_{y}^{k}(t)\right], m, k=0, \ldots, K-1$, given by

$$
\left[\widetilde{S}_{x}^{k}(t), \widetilde{S}_{y}^{k}(t)\right] \approx\left[e_{x}, e_{y}\right] \cdot \tilde{s}(t) p_{t}^{k}(u)+\left[N_{x}^{k}(t), N_{y}^{k}(t)\right]
$$

where

$$
p_{t}^{k}(u)=e^{j\left[k\left(u-\frac{2 \pi t}{L}\right)+(N-K)\left(\frac{u}{2}-\frac{\pi t}{L}\right)\right]} \frac{\sin \left(\frac{N u}{2}-\frac{N \pi t}{L}\right)}{\sin \left(\frac{K u}{2}-\frac{K \pi t}{L}\right)}
$$

and $\left[N_{x}^{k}(t), N_{y}^{k}(t)\right]$ are the $K$-point IDFTs of $\left[n_{x}^{m}(t), n_{y}^{m}(t)\right] e^{-j m \widehat{N u}}, m, k=0, \ldots, K-1$.

We compute the differential signals between any two adjacent IDFT outputs, $\left[\left(\widetilde{S}_{x}^{k}(t)\right)^{*} \widetilde{S}_{x}^{k+1}(t),\left(\widetilde{S}_{y}^{k}(t)\right)^{*} \widetilde{S}_{y}^{k+1}(t)\right]$, to obtain an estimate of $u$. The differential signals, denoted by 
$\left[d_{x}^{k}(t), d_{y}^{k}(t)\right], k=0, \ldots, K-2$, can be expressed as

$$
\begin{aligned}
{\left[d_{x}^{k}(t), d_{y}^{k}(t)\right]=} & \underbrace{\left[\left|e_{x}\right|^{2},\left|e_{y}\right|^{2}\right]|\tilde{s}(t)|^{2}\left|\frac{\sin \left(\frac{N u}{2}-\frac{N \pi t}{L}\right)}{\sin \left(\frac{K u}{2}-\frac{K \pi t}{L}\right)}\right|^{2} e^{j\left(u-\frac{2 \pi t}{L}\right)}}_{\text {signal components }} \\
& +\left[\widetilde{N}_{x}^{k}(t), \widetilde{N}_{y}^{k}(t)\right],
\end{aligned}
$$

where

$$
\begin{aligned}
\tilde{N}_{l}^{k}(t)= & \left(N_{l}^{k}(t)\right)^{*} N_{l}^{k+1}(t)+e_{l}^{*} \tilde{s}^{*}(t)\left(p_{t}^{k}(u)\right)^{*} N_{l}^{k+1}(t) \\
& +e_{l} \tilde{s}(t) p_{t}^{k+1}(u)\left(N_{l}^{k}(t)\right)^{*}, \quad l \in\{x, y\}
\end{aligned}
$$

can be approximated as complex Gaussian noises with zero means, and noise powers $\sigma_{\widetilde{N}_{l}}^{2}=2\left|e_{l}\right|^{2}|\tilde{s}(t)|^{2}\left|p_{t}^{k}(u)\right|^{2} \sigma_{N_{l}}^{2} \cdot \sigma_{N_{l}}^{2}$ denotes noise power of $N_{l}^{k}(t)(l \in\{x, y\})$ given by $\sigma_{N_{l}}^{2}=$ $\sigma_{n}^{2} / K$. It is seen from (11) that the estimate of $u, \hat{u}$, can be unambiguously obtained by $\hat{u}=\arg \left\{\left(d_{x}^{k}(t)+d_{y}^{k}(t)\right) e^{\frac{j 2 \pi t}{L}}\right\}$. Likewise, $\left[d_{x}^{k}(t), d_{y}^{k}(t)\right]$ over all subarrays and symbols can be merged to improve the accuracy of $\hat{u}$.

Unlike the Algorithm 2 in [12], we propose to use $\hat{u}$ to update $\widehat{N u}$, i.e., $\widehat{N u} \leftarrow N \hat{u}$, and in turn to re-estimate $u$. As a result, the estimation accuracy of $u$ is improved after one iteration due to the upgraded $\widehat{N u}$. The improved MSE performance of $\widehat{N u}$ and the resulting $\hat{u}$ will be demonstrated in the simulation results. The enhanced AoA estimation algorithm is summarized in Algorithm I, where $\left(\tilde{\mathbf{S}}_{l}^{q}(t)\right)_{\left(k_{1}: k_{2}\right)}$ denotes the vector consisting of the $k_{1}$ th to $k_{2}$ th elements of $\tilde{\mathbf{S}}_{l}^{q}(t)$ and $(\cdot)^{T}$ represents the transpose of $(\cdot)$.

\section{Extension to Uniform Planar Array}

The proposed algorithm can be readily extended from uniform linear arrays to uniform planar arrays. Let $M_{x}$ and $M_{y}$ denote the numbers of subarrays along $x$ - and $y$-axis directions, and $N_{x}$ and $N_{y}$, the numbers of EMVS in a subarray along the two directions. At the $t$ th symbol, the $\left(n_{x}\right.$, $\left.n_{y}\right)$ th $\left(n_{x}=0, \ldots, N_{x}-1 ; n_{y}=0, \ldots, N_{y}-1\right)$ phase shift of the $\left(m_{x}, m_{y}\right)$ th $\left(m_{x}=0, \ldots, M_{x}-1 ; m_{y}=0, \ldots, M_{y}-1\right)$ subarray is given by

$$
\begin{aligned}
\alpha_{t}^{m_{x}, m_{y}}\left(n_{x}, n_{y}\right)= & -2 \pi n_{x}\left(\bmod \left\{m_{x}, K_{x}\right\} / K_{x}+t / L_{x}\right) \\
& -2 \pi n_{y}\left(\bmod \left\{m_{y}, K_{y}\right\} / K_{y}+t / L_{y}\right),
\end{aligned}
$$

and the associated radiation pattern is

$$
\begin{aligned}
P_{t}^{m_{x}, m_{y}}\left(u_{x}, u_{y}\right)= & e^{j\left(N_{x}-1\right) \omega_{x, t}^{m_{x}}} \frac{\sin \left(N_{x} \omega_{x, t}^{m_{x}}\right)}{\sin \left(\omega_{x, t}^{m_{x}}\right)} \\
& \cdot e^{j\left(N_{y}-1\right) \omega_{y, t}^{m_{y}}} \frac{\sin \left(N_{y} \omega_{y, t}^{m_{y}}\right)}{\sin \left(\omega_{y, t}^{m_{y}}\right)},
\end{aligned}
$$

where $u_{x}=\frac{2 \pi}{\lambda} d \sin \theta \cos \phi, u_{y}=\frac{2 \pi}{\lambda} d \sin \theta \sin \phi$ and $\phi$ denotes the azimuth angle of the received signal; $\omega_{x, t}^{m_{x}}=$ $\frac{u_{x}}{2}-\pi\left(\frac{m_{x}}{M_{x}}+\frac{t}{L_{x}}\right)$ and $\omega_{y, t}^{m_{y}}=\frac{u_{y}}{2}-\pi\left(\frac{m_{y}}{M_{y}}+\frac{t}{L_{y}}\right)$ assuming $K_{x}=M_{x}$ and $K_{y}=M_{y} ;\left(K_{x}, K_{y}\right)$ and $\left(L_{x}, L_{y}\right)$ are the extensions of $K$ and $L$ to the $x$ - and $y$-axis directions, respectively.
For the subarrays along $x$-axis direction, the corresponding $G_{t}^{m_{x}}\left(u_{x}, u_{y}\right)$ in (6) is given by

$$
\begin{aligned}
& G_{t}^{m_{x}}\left(u_{x}, u_{y}\right) \\
= & \left(P_{t}^{m_{x}, m_{y}}\left(u_{x}, u_{y}\right)\right)^{*} P_{t}^{m_{x}+1, m_{y}}\left(u_{x}, u_{y}\right) \\
= & e^{\frac{-j\left(N_{x}-1\right) \pi}{K_{x}}} \frac{(-1)^{Q_{x}} \sin ^{2}\left(N_{x} \omega_{x, t}^{m_{x}}\right)}{\sin \left(\omega_{x, t}^{m_{x}}\right) \sin \left(\omega_{x, t}^{m_{x}+1}\right)} \cdot\left|\frac{\sin \left(N_{y} \omega_{y, t}^{m_{y}}\right)}{\sin \left(\omega_{y, t}^{m_{y}}\right)}\right|^{2},
\end{aligned}
$$

where $Q_{x}$ is the extension of $Q$ to the $x$-direction. It is observed from (14) that since $\left|\frac{\sin \left(N_{y} \omega_{y, t}^{m_{y}}\right)}{\sin \left(\omega_{y, t}^{m}\right)}\right|^{2}>0$ assuming $\frac{\sin \left(N_{y} \omega_{y, t}^{m_{y}}\right)}{\sin \left(\omega_{y, t}^{m y}\right)} \neq 0$, the Theorem 1 [12] is still applicable here, i.e.,

$$
m_{x}^{\prime}=\underset{m_{x}=0: K_{x}-1}{\operatorname{argmax}}\left\{\left|\sum_{m_{y}=0}^{M_{y}-1}\left(\rho_{x}^{m_{x}}(t)+\rho_{y}^{m_{x}}(t)\right)\right|\right\},
$$

where $\rho_{x}^{m_{x}}(t)$ and $\rho_{y}^{m_{x}}(t)$ denote the differential signals between the output signals of the $\left(m_{x}, m_{y}\right)$ th and $\left(m_{x}+1\right.$, $m_{y}$ )th subarrays for $x$ - and $y$-axis dipoles, respectively. All available subarray outputs along $y$-axis direction are coherently combined for finding $m_{x}^{\prime}$ in order to improve the SNR of the differential signals. Given $m_{x}^{\prime}$, the signs of $\left[\rho_{x}^{m_{x}}(t), \rho_{y}^{m_{x}}(t)\right]$ can be aligned following (8) as $\left[\tilde{\rho}_{x}^{m_{x}}(t), \tilde{\rho}_{y}^{m_{x}}(t)\right]$. As a result, $\widehat{N_{x} u_{x}}$ can be obtained by

$\widehat{N_{x} u_{x}}=\arg \left\{e^{\frac{j\left(N_{x}-1\right) \pi}{K}} \sum_{t=0}^{T-1} \sum_{m_{y}=0}^{M_{y}-1} \sum_{m_{x}=0}^{M_{x}-2}\left(\tilde{\rho}_{x}^{m_{x}}(t)+\tilde{\rho}_{y}^{m_{x}}(t)\right)\right\}$.

Likewise, we have $\widehat{N_{y} u_{y}}$ by determining $m_{y}^{\prime}$. Given $\widehat{N_{x} u_{x}}$ and $\widehat{N_{y} u_{y}}$, we can estimate $u_{x}$ and $u_{y}$ unambiguously following a similar way to the estimation of $u$ in Algorithm I.

\section{AoA Estimation Performance Evaluation}

In the following, we evaluate the performance of the proposed algorithm using the MSEs of $\hat{u}$, and derive its closedform MSELBs. The ideal case for $\hat{u}$ occurs when $\widehat{N u}=N u$, i.e., $e^{j m(N u-\widehat{N u})}=1$. This corresponds to the case where the output signals of all subarrays are perfectly aligned. From (11) and Step 22 in Algorithm I, the estimation of $u$ is formulated as the phase estimation of the accumulation of $d_{x}^{q K+k}(t)+d_{y}^{q K+k}(t)$ over all $k, q$ and $t$ (denoted by $D$ ), given $\tilde{s}(t), u, \gamma$ and $\eta$ (collectively denoted by $\mathbf{c}$ ). For convenience of analysis, consider $M=K$, and hence $D$ is complex Gaussian distributed with conditional mean

$$
m_{D}=\left(\left|e_{x}\right|^{2}+\left|e_{y}\right|^{2}\right) \sum_{t=0}^{T-1 K-2} \sum_{k=0}|\tilde{s}(t)|^{2}\left|p_{t}^{k}(u)\right|^{2} e^{j\left(u-\frac{2 \pi t}{L}\right)}
$$

and conditional variance

$$
\begin{aligned}
\sigma_{D}^{2} & =\sum_{t=0}^{T-1} \sum_{k=0}^{K-2}\left(\sigma_{\widetilde{N}_{x}}^{2}+\sigma_{\widetilde{N}_{y}}^{2}\right) \\
& =\frac{2\left(\left|e_{x}\right|^{2}+\left|e_{y}\right|^{2}\right) \sigma_{n}^{2}}{K} \sum_{t=0}^{T-1} \sum_{k=0}^{K-2}|\tilde{s}(t)|^{2}\left|p_{t}^{k}(u)\right|^{2} .
\end{aligned}
$$


As a result, the conditional SNR of $D$ given $\mathbf{c}, \gamma_{\mathbf{c}}$, is given by

$$
\gamma_{\mathbf{c}}=\frac{\left|m_{D}\right|^{2}}{\sigma_{D}^{2}}=\frac{K\left(\left|e_{x}\right|^{2}+\left|e_{y}\right|^{2}\right)}{2 \sigma_{n}^{2}} \sum_{t=0}^{T-1} \sum_{k=0}^{K-2}|\tilde{s}(t)|^{2}\left|p_{t}^{k}(u)\right|^{2} .
$$

Taking the expectation of $\gamma_{\mathbf{c}}$ over $\mathbf{c}$ and assuming $d=\lambda / 2$, we have the average SNR of $D, \bar{\gamma}$, as

$$
\begin{aligned}
\bar{\gamma}= & \frac{K \gamma_{s}}{2} \sum_{t=0}^{T-1} \sum_{k=0}^{K-2} \mathbb{E}_{u, \gamma}\left\{\left|p_{t}^{k}(u)\right|^{2}\left(\sin ^{2} \gamma\left(1-u^{2} / \pi^{2}\right)+\cos ^{2} \gamma\right)\right\} \\
= & \frac{K \gamma_{s}}{2} \sum_{t=0}^{T-1} \sum_{k=0}^{K-2} \mathbb{E}_{u}\left\{\left|p_{t}^{k}(u)\right|^{2}\right\} \\
& -\frac{K \gamma_{s}}{2 \pi^{2}} \sum_{t=0}^{T-1} \sum_{k=0}^{K-2} \mathbb{E}_{\gamma}\left\{\sin ^{2} \gamma\right\} \cdot \mathbb{E}_{u}\left\{\left|u p_{t}^{k}(u)\right|^{2}\right\} \\
\stackrel{(a)}{=} & \frac{N T(K-1) \gamma_{s}}{2}-\frac{K(K-1) \gamma_{s}}{8 \pi^{3}} \\
& \cdot \sum_{t=0}^{T-1} \int_{-\pi}^{\pi}\left|\frac{u \sin \left(\frac{N u}{2}-\frac{N \pi t}{L}\right)}{\sin \left(\frac{K u}{2}-\frac{K \pi t}{L}\right)}\right|^{2} \mathrm{~d} u
\end{aligned}
$$

where $\gamma_{s}=\mathbb{E}\left\{\frac{|\tilde{s}(t)|^{2}}{\sigma_{n}^{2}}\right\}$. (a) holds because

$$
\mathbb{E}_{u}\left\{\left|p_{t}^{k}(u)\right|^{2}\right\}=\frac{1}{2 \pi} \int_{-\pi}^{\pi}\left|\frac{\sin \left(\frac{N u}{2}-\frac{N \pi t}{L}\right)}{\sin \left(\frac{K u}{2}-\frac{K \pi t}{L}\right)}\right|^{2} \mathrm{~d} u=N / K
$$

by assuming $u$ uniformly distributed within $[-\pi, \pi)$, denoted by $u \sim \mathbf{U}(-\pi, \pi)$. We also assume $\gamma \sim \mathbf{U}(0, \pi / 2)$ in (19).

Denote the probability density function (pdf) of $\hat{u}$ as $f_{\hat{u}}(\hat{u})$. Assuming that $|\tilde{s}(t)|$ is Rayleigh distributed, we have $f_{\hat{u}}(\hat{u})=$ $f_{1}(\hat{u}, \bar{\gamma})$. At high SNRs, $f_{1}(\hat{u}, \bar{\gamma})$ is approximated as [8]

$$
f_{1}(\hat{u}, \bar{\gamma}) \approx \frac{\sqrt{\bar{\gamma} \pi^{2}+1}}{2 \pi\left(\bar{\gamma} \hat{u}^{2}+1\right)^{3 / 2}}, \quad-\pi \leq \hat{u}<\pi .
$$

If $\left|p_{t}^{k}(u)\right|$ achieves the maximum value $Q, f_{1}(\hat{u}, \bar{\gamma})$ will be the true pdf of $\hat{u}$. However, since $\left|p_{t}^{k}(u)\right| \leq Q, \forall k, t, u$, there will be an SNR reduction. Moreover, our derivation is based on the assumption of $\widehat{N u}=N u$. As a result, the actual MSE, $\sigma_{\hat{u}}^{2}$, will always be greater than that calculated using $f_{1}(\hat{u}, \bar{\gamma})$, i.e.,

$$
\begin{aligned}
\sigma_{\hat{u}}^{2} & \geq \int_{-\pi}^{\pi} \hat{u}^{2} f_{1}(\hat{u}, \bar{\gamma}) d \hat{u} \\
& =\frac{\sqrt{\bar{\gamma} \pi^{2}+1}}{\pi \bar{\gamma}^{3 / 2}} \sinh ^{-1}(\sqrt{\bar{\gamma}} \pi)-\frac{1}{\bar{\gamma}}=\text { MSELB. }
\end{aligned}
$$

This MSELB is asymptotically tight as $T$ and $K$ increase. Similarly, when the signal reception is performed with a single-polarized hybrid array, the average SNRs of $D$ using $x$ - or $y$-axis dipoles only are given by $\bar{\gamma}_{x}=\bar{\gamma}-\bar{\gamma}_{y}$ and $\bar{\gamma}_{y}=\frac{N T(K-1) \gamma_{s}}{4}$, respectively. By substituting them into (22), it can be verified that the MSELB in [12] is a special case of our results.

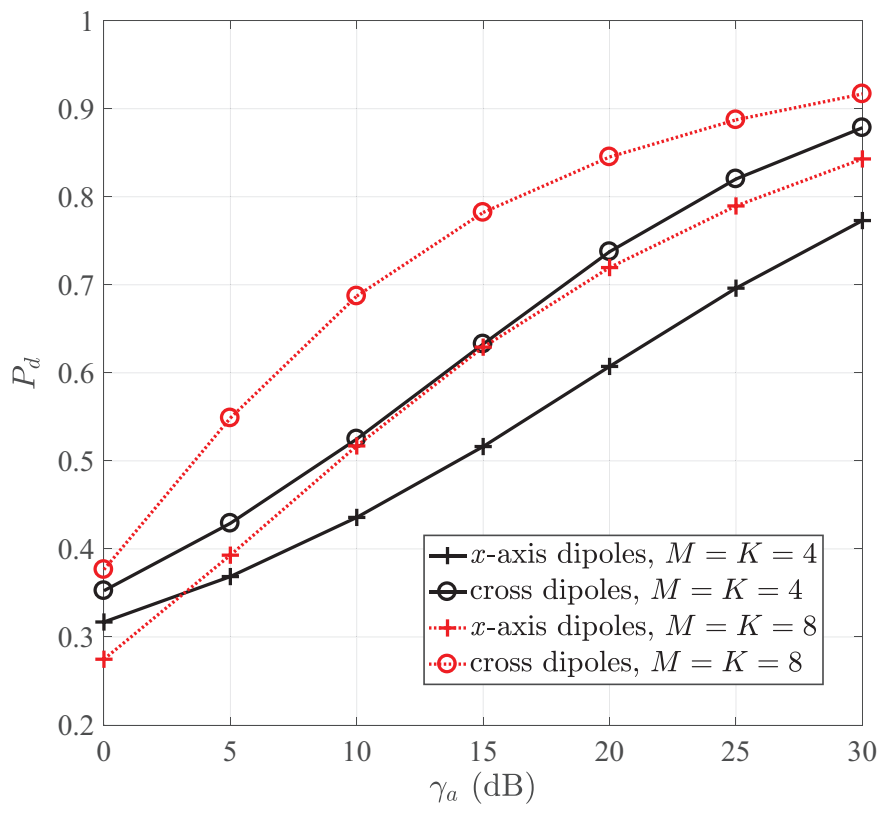

Fig. 2. Detection probability of $m^{\prime}, P_{d}$ versus $\gamma_{a}$, where $N=8$ and $T=4$.

\section{Simulation Results}

In this section, we present the simulation results to evaluate the proposed algorithm and analysis using hybrid dualpolarized arrays. We also simulate the state of the art [12] using hybrid single-polarized arrays for comparison. Denote the average SNR per EMVS as $\gamma_{a}$, which is given by $\gamma_{a}=N \gamma_{s}$. The reference signals, $\tilde{s}(t)$, are generated following complex Gaussian distributions. Considering the reception of polarized signals following $u \sim \mathbf{U}(-\pi, \pi), \gamma \sim \mathbf{U}(0, \pi / 2)$ and $\eta \sim \mathbf{U}(-\pi, \pi)$, simulation results are obtained by averaging 50000 independent trials.

Fig. 2 compares the detection probability of $m^{\prime}, P_{d}$, versus $\gamma_{a}$ using dual-polarized and single-polarized ( $x$-axis dipoles) arrays, where the number of EMVSs in each subarray, $N$, is fixed to be 8 and the number of reference signals, $T$, is set to be 4 . As shown in the figure, the dual-polarized array outperforms the single-polarized one in the state of the art in terms of $P_{d}$, which is defined as the probability of correctly finding the index of differential signals with the largest amplitude. It indicates that the use of dual-polarized arrays is better on calibrating the signs of differential signals and thus improves the accuracy of $\widehat{N u}$. Also, using more subarrays results in higher detection probability as $\gamma_{a}$ increases.

The MSEs of the estimates are shown as a function of $\gamma_{a}$ in Fig. 3, where $N=8$ and $M=K=T=4$. It can be seen that the MSEs of both $e^{j \widehat{N u}}$ (refer to Step 10) and $\hat{u}_{1}$ (refer to initial estimate at Step 22) in Algorithm I using dual-polarized arrays (plus sign marker) are lower than those using singlepolarized arrays (diamond marker), which are in line with the results in Fig. 2. Along with the update of $\widehat{N u}$ (red plus sign marker) to be $N \widehat{u}_{1}$ (red circle marker), its MSE performance is improved by up to $5 \mathrm{~dB}$ at the MSE of 0.1. It is also shown that a resulting $2 \mathrm{~dB}$ improvement of AoA estimation from $\hat{u}_{1}$ 


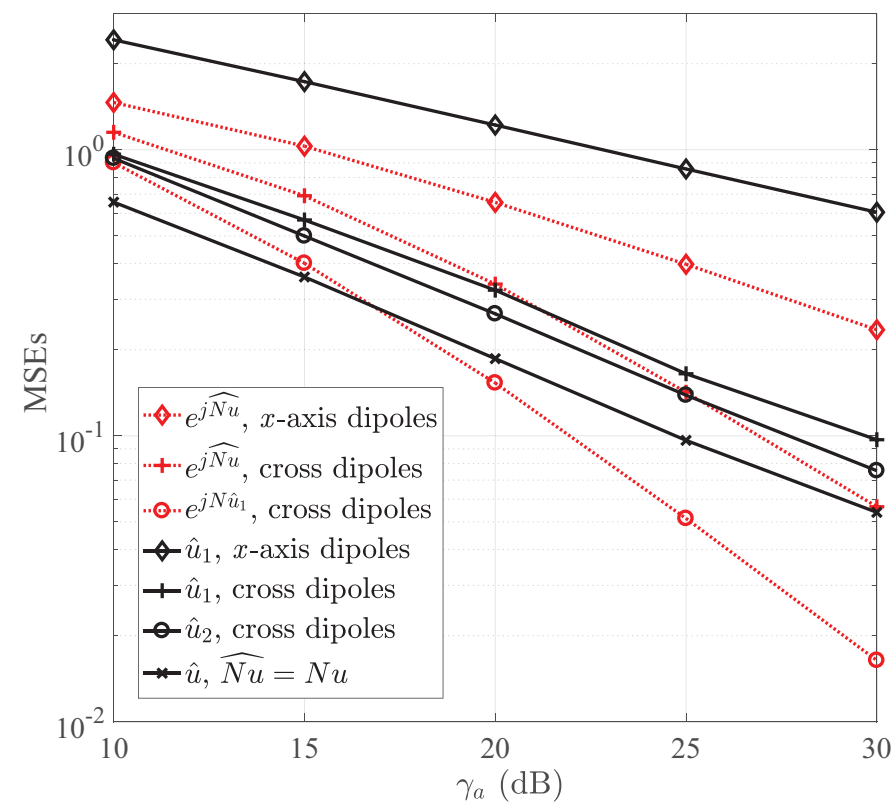

Fig. 3. The MSEs under different scenarios versus $\gamma_{a}$, where $N=8$ and $M=K=T=4$.

(black plus sign marker) to $\hat{u}_{2}$ (black circle marker and refer to the updated estimate at Step 23) can be achieved because of the improved $\widehat{N u}$. The MSEs of $\hat{u}$ (cross marker) under the assumption that $\widehat{N u}=N u$, i.e., the output signals of all subarrays are perfectly aligned, are displayed for comparison.

The simulated MSEs and calculated MSELBs using dualpolarized and single-polarized ( $x / y$-axis dipoles) arrays under different numbers of reference signals are respectively plotted in Fig. 4, where $\gamma_{a}$ is fixed to be $0 \mathrm{~dB}, N=16$ and $M=K=$ 8. It is seen from Fig. 4 that the MSELBs become increasingly tight to the proposed algorithm using dual-polarized arrays as the number of reference signals increases. The gaps between the simulated MSEs and MSELBs for the proposed one are closer than those in [12].

\section{CONCLUSION}

An enhanced AoA estimation algorithm using localized hybrid dual-polarized arrays is developed to improve the AoA estimation accuracy for a polarized mmWave signal. Employing the polarization diversity, dual-polarized antennas can effectively enhance the calibration capability of the signs of differential signals, and thus the SNR for AoA estimation. Simulation results of MSEs show that, through updating the estimate of phase offset between adjacent subarrays, the proposed algorithm is able to dramatically improve the AoA estimation performance.

\section{REFERENCES}

[1] R. W. Heath, Jr., N. Gonzalez-Prelcic, S. Rangan, W. Roh, and A. M. Sayeed, "An overview of signal processing techniques for millimeter wave MIMO systems," IEEE J. Sel. Topics Signal Process., vol. 10, no. 3, pp. 436-453, Apr. 2016.

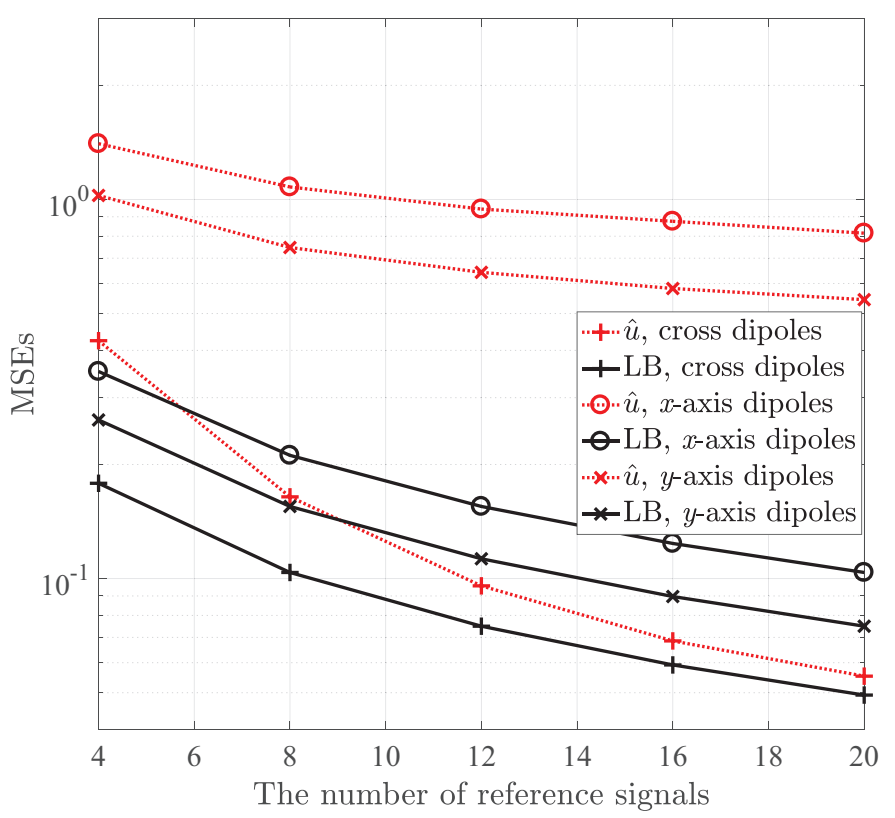

Fig. 4. Simulated MSEs and MSELBs versus the number of reference signals, where $\gamma_{a}=0 \mathrm{~dB}, N=16$ and $M=K=8$.

[2] A. L. Swindlehurst, E. Ayanoglu, P. Heydari, and F. Capolino, "Millimeter-wave massive MIMO: The next wireless revolution?" IEEE Commun. Mag., vol. 52, no. 9, pp. 56-62, Sep. 2014.

[3] F. Sohrabi and W. Yu, "Hybrid digital and analog beamforming design for large-scale antenna arrays," IEEE J. Sel. Topics Signal Process., vol. 10, no. 3, pp. 501-513, Apr. 2016.

[4] J. Zhang, X. Huang, V. Dyadyuk, and Y. Guo, "Massive hybrid antenna array for millimeter-wave cellular communications," IEEE Wireless Commun., vol. 22, no. 1, pp. 79-87, Feb. 2015.

[5] S. X. Ta, I. Park, and R. W. Ziolkowski, "Crossed dipole antennas: A review," IEEE Antennas Propag. Mag., vol. 57, no. 5, pp. 107-122, Oct. 2015.

[6] J. Song, J. Choi, S. G. Larew, D. J. Love, T. A. Thomas, and A. Ghosh, "Adaptive millimeter wave beam alignment for dual-polarized MIMO systems," IEEE Trans. Wireless Commun., vol. 14, no. 11, pp. 6283-6296, Nov. 2015.

[7] O. Jo, J.-J. Kim, J. Yoon, D. Choi, and W. Hong, "Exploitation of dual-polarization diversity for $5 \mathrm{G}$ millimeter-wave MIMO beamforming systems," IEEE Trans. Antennas Propag., vol. 65, no. 12, pp. 6646-6655, Dec. 2017.

[8] X. Huang, Y. J. Guo, and J. Bunton, "A hybrid adaptive antenna array," IEEE Trans. Wireless Commun., vol. 9, no. 5, pp. 1770-1779, May 2010.

[9] J. A. Zhang, X. Huang, and Y. J. Guo, "Adaptive searching and tracking algorithm for AoA estimation in localized hybrid array," in Proc. IEEE Int. Conf. Commun. Workshop (ICCW), Jun. 2015, pp. 1095-1100.

[10] X. Huang and Y. J. Guo, "Frequency-domain AoA estimation and beamforming with wideband hybrid arrays," IEEE Trans. Wireless Commun., vol. 10, no. 8, pp. 2543-2553, Aug. 2011.

[11] J. A. Zhang, W. Ni, P. Cheng, and Y. Lu, "Angle-of-arrival estimation using different phase shifts across subarrays in localized hybrid arrays," IEEE Commun. Lett., vol. 20, no. 11, pp. 2205-2208, Nov. 2016.

[12] K. Wu, W. Ni, T. Su, R. Liu, and Y. J. Guo, "Robust unambiguous estimation of angle-of-arrival in hybrid array with localized analog subarrays," IEEE Trans. Wireless Commun., vol. 17, no. 5, pp. 29873002, May 2018

[13] K. Wu, W. Ni, T. Su, R. Liu, and Y. J. Guo, "Fast and accurate estimation of angle-of-arrival for satellite-borne wideband communication system," IEEE J. Sel. Areas Commun., vol. 36, no. 2, pp. 314-326, Feb. 2018.

[14] L. Wan, K. Liu, Y. Liang, and T. Zhu, "DOA and polarization estimation for non-circular signals in 3-D millimeter wave polarized massive MIMO systems," Dec. 2017. [Online]. Available: https://arxiv.org/abs/1712.05587 